\title{
Propagation of privileged waves in longitudinally inhomogeneous medium with linear birefringence and dichroism
}

\author{
M.S. Koev* ${ }^{*}$ I.S. Kolomiets, S.N. Savenkov, Ye.A. Oberemok, Yu.A. Skoblya \\ Taras Shevchenko Kyiv National University, \\ 4, Glushkov Ave, 03127 Kyiv, Ukraine \\ Phone: +38(044)526-0570, fax: +38(044) 526-1073 \\ *E-mail: kolomiets55@gmail.com
}

\begin{abstract}
Anisotropic properties of longitudinally inhomogeneous medium with linear birefringence and dichroism were considered. Differential Jones matrix model for this class of media was obtained. The equation for the polarization complex variable that describes the evolution of polarization state in this class of media was found. Partial solutions describing evolution of the privileged polarization states were obtained. The features of privileged polarization states and the conditions of their existence in this class of media were analyzed.
\end{abstract}

Keywords: Jones matrix, birefringence, dichroism, privileged polarization states.

Manuscript received 02.04.14; revised version received 20.08.14; accepted for publication 29.10.14; published online 10.11.14.

\section{Introduction}

The history of investigation of inhomogeneous nondepolarizing media returns us to [1]. This problem was solved using the Jones matrix methods [2]. In [3] at first the Jones matrix for longitudinal inhomogeneous anisotropic medium was presented. Next step was made by R.M.A. Azzam in [4] where he considered the type of longitudinal inhomogeneous medium with linear birefringence. Considered in particular in [4] were examples of these medium. It was cholesteric liquid crystals and twisted nematics. Development of modern display technology led to the fact that these types of liquid crystals very widely used $[5,6]$. Therefore, a number of the next works [7-9] dealt with the features of anisotropy formation in longitudinally inhomogeneous medium with linear birefringence, and in the more general case of elliptical birefringence. However, as we know [10] for certain wavelengths in different types of cholesteric liquid crystals and twisted nematics, besides linear birefringence occurs as linear dichroism. One of the main features of the anisotropy arising in longitudinally inhomogeneous twisted media are waves with privileged polarization states, which propagate in this media as in the optically active media. To study the features of this polarization in longitudinal inhomogeneous media with linear birefringence and dichroism is the main goal of this work.

\section{The structure of medium}

Non-depolarizing longitudinal inhomogeneous medium with linear birefringence and dichroism can be equivalently represented as a sequence of molecular planes that consists of molecules oriented parallel to each other [4]. Each this molecular plane can be considered as a sufficiently thin layer (thickness of this layer is considered to be much smaller than the size of the medium in the direction of light propagation) with linear birefringence and dichroism. In general, the axis 
of birefringence and dichroism in these molecules can differ. Thus, each molecule is characterized by two axes: the fast axis (axis of birefringence) and the axis of least absorption (dichroism axis) lying in the plane of each molecular layer. In this media, at propagation in the direction perpendicular to the molecular planes the direction of anisotropy axis of each molecular layer returns (twisted) compared to the previous one by some angle. Consequently, the resulting sequence of molecular planes is the object with linear birefringence and dichroism that form longitudinally (along the direction of light propagation, which coincides with the direction around which twisting occurs) non-depolarizing inhomogeneous medium with its linear birefringence and dichroism. When considering propagation of polarized light along the axis $z$ of the Cartesian rectangular coordinate system, which coincides with the axis of twist of the layered medium (Fig. 1), the azimuth of birefringence $\alpha$ and dichroism $\theta$ axis of molecular plane at a distance $z$ from the input molecular plane are defined as:

$\alpha=\alpha_{0} z, \theta=\theta_{0} z$,

where $\alpha_{0}, \theta_{0}$ are the specific values of orientation of the birefringence and dichroism axis per unity thickness, defined as:

$\alpha_{0}=\frac{2 \pi}{p}, \theta_{0}=\frac{2 \pi}{p}$,

where $p$ is the step of a helical structure of medium (the smallest distance between the planes with the same orientation of molecules).

\section{Polarimetric models of media}

Anisotropic properties of one molecular layer in this medium are described by the differential Jones matrix (in circular basis) that includes parameters of birefringence and dichroism [3]:

$N=\left[\begin{array}{cc}0 & \frac{1}{2}\left(i \delta_{0} e^{2 i \alpha_{0} z}+\xi_{0}^{2 i \theta_{0} z}\right) \\ \frac{1}{2}\left(i \delta_{0} e^{-2 i \alpha_{0} z}+\xi_{0}^{-2 i \theta_{0} z}\right) & 0\end{array}\right]$.

As it is known [11], the Jones vector contains information about the state of light polarization, absolute amplitude (intensity) and phase of the light wave. But in the case when there is only relevant information about the state of polarization, more convenient is a complex variable $\chi$, defined as:

$\chi=\frac{E_{y}}{E_{x}}$,

where $E_{x}, E_{y}$ are the projections of the Jones vector on the $x$ and $y$ axes in the Cartesian rectangular coordinate system, respectively. To describe evolution of the state of polarization in anisotropic media, one can use the scalar differential equation for the complex variable $[4,11]$ :

$$
\frac{d \chi}{d z}=-N_{12} \chi^{2}+\left(N_{22}-N_{11}\right) \chi+N_{21}
$$

where $N_{i, j}$ are elements of the differential Jones matrix (3) that describes the properties of medium where light propagates. Substituting the Jones matrix for a given class of media (3) into Eq. (5), we get:

$$
\frac{d \chi}{d z}=\frac{1}{2}\left(i \delta_{0} e^{-2 i \alpha_{0} z}+\xi_{0}^{-2 i \theta_{0} z}-\left(i \delta_{0} e^{2 i \alpha_{0} z}+\xi_{0}^{2 i \theta_{0} z}\right) \chi^{2}\right) .
$$

As shown in $[4,11]$ in longitudinally inhomogeneous media, there can exist privileged waves that propagate in it like to that in medium with optical activity (azimuth changes linearly with the coordinate $z$ from one molecular layer to another, and the ellipticity angle does not change). The complex variables $K_{1,2}$ that describe the state of polarization of these waves are a particular solution of Eq. (5) and are sought in the form:

$\chi_{1,2}=K_{1,2} e^{-2 i \gamma_{0} z}$,

where $2 \gamma_{0}$ is the doubled specific angle of rotation of polarization plane inherent to the privileged waves in one molecular layer, $\chi_{1,2}$ are complex variables describing evolution of the polarization states of privileged waves in this class of media. Substituting Eq. (7) into Eq. (6), we find that partial solutions in the form of polarizations of privileged wave can exist in a given class of media only if

$\alpha_{0}=\theta_{0}$.

That is, orientations of the axes of birefringence and dichroism in each molecular layer coincide. So, let us consider the case (8). When performing the resulting conditions for existence of the privileged wave, Fig. 1 becomes similar to that shown in Fig. 2.

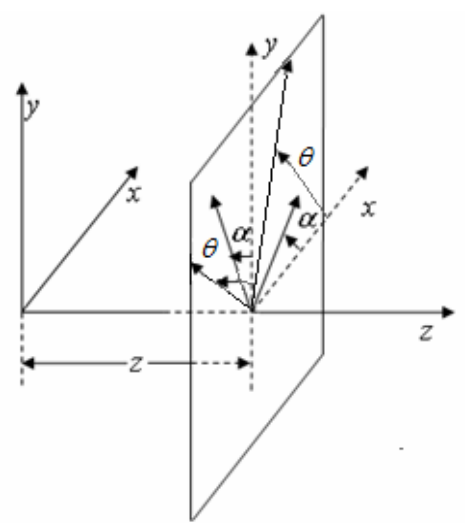

Fig. 1. Molecular plane of longitudinally inhomogeneous medium at a distance $z$ from the input plane. The case $\alpha_{0} \neq \theta_{0}$. 


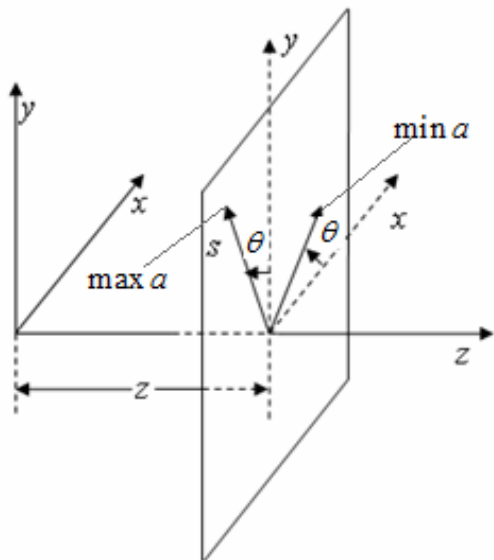

Fig. 2. Molecular plane of longitudinally inhomogeneous medium at a distance $z$ from the input plane. The case $\alpha_{0} \neq \theta_{0}$.

As a result, the axis of birefringence and dichroism can be found using the second relation in Eq. (1), and each molecule in the molecular plane has an elongated shape, and there are appropriate mechanisms for linear anisotropy in this class of media.

In the case $\alpha_{0}=\theta_{0}$, the anisotropic properties of one molecular layer in this medium are described by the differential Jones matrix (in circular basis):

$$
N=\left[\begin{array}{cc}
0 & \frac{1}{2}\left(i \delta_{0}+\xi_{0}\right) e^{2 i \theta_{0} z} \\
\frac{1}{2}\left(i \delta_{0}+\xi_{0}\right) e^{-2 i \theta_{0} z} & 0
\end{array}\right] .
$$

\section{Evolution of privileged waves}

In the case $\alpha_{0}=\theta_{0}$, Eq. (6) describing evolution of polarization states in anisotropic medium takes the following form:

$$
\frac{d \chi}{d z}=\frac{1}{2} e^{2 i \theta_{0} z}\left(i \delta_{0}+\xi_{0}\right)\left(\chi^{2} e^{4 i \theta_{0} z}-1\right) .
$$

Eq. (10) has a partial solution in the form:

$$
\chi_{1,2}=K_{1,2} e^{-2 i \theta_{0} z} \text {, }
$$

where $K_{1,2}$ are the roots of the characteristic equation for (10) and describe the complex variables of polarization of privileged waves:

$$
K_{1,2}=\frac{2 \theta}{\delta_{0}-i \xi_{0}} \mp \sqrt{\left(\frac{2 \theta}{\delta_{0}-i \xi_{0}}\right)^{2}+1} .
$$

To find the main characteristics of the polarization states inherent to privileged waves, such as the azimuth $\theta_{1,2}$ and angles of ellipticity $e_{1,2}$, we used the relation [11]:

$$
\begin{aligned}
& \theta_{1,2}=\frac{1}{2} \operatorname{Arg}\left(K_{1,2}\right), \\
& e_{1,2}=\frac{1}{2} \operatorname{Arctan}\left(\frac{\left|K_{1,2}\right|-1}{\left|K_{1,2}\right|+1}\right) .
\end{aligned}
$$

Substituting (12) in (13), (14), we get:

$$
\begin{aligned}
& \theta_{1,2}=\frac{1}{2} \operatorname{Arg}\left(\mp \sqrt{\frac{4 \theta_{0}^{2}}{\left(\delta_{0}-i \xi_{0}\right)^{2}}+1}+\frac{2 \theta_{0}}{\delta_{0}-i \xi_{0}}\right), \\
& e_{1,2}=\operatorname{Arctan}\left(1-\frac{2}{1+\left|\sqrt{\frac{4 \theta_{0}^{2}}{\left(\delta_{0}-i \xi_{0}\right)^{2}}+1} \mp \frac{2 \theta_{0}}{\delta_{0}-i \xi_{0}}\right|}\right) .
\end{aligned}
$$

Being based on Eq. (15), it should be noted that in contrast to the class of media in the absence of dichroism $[8,9]$ the azimuths of polarization orientation of privileged waves do not coincide with the respective orientations of the molecular planes. Analyzing Eq. (16), we find that polarizations of privileged waves are elliptical. It is interesting to note that $e_{1,2}=0$ only if $\theta_{0}=0$, i.e. when this medium is homogeneous.

Now examine polarization of privileged waves on orthogonality. To do it, we substitute (12) in the product $K_{1} K_{2}{ }^{*}\left(K_{1}\right.$ and $K_{2}$ are multiplied by $z / z$ and moved from the specific to the integral anisotropy parameters based on the equations $\left.P=-\ln \left(\xi_{0} z\right), \delta=\delta_{0} z, \theta=\theta_{0} z\right)$, which should give -1 for the case of orthogonal corresponding polarization states. Real and imaginary parts of the obtained product $K_{1} K_{2}{ }^{*}$ are shown in Fig. 3 .
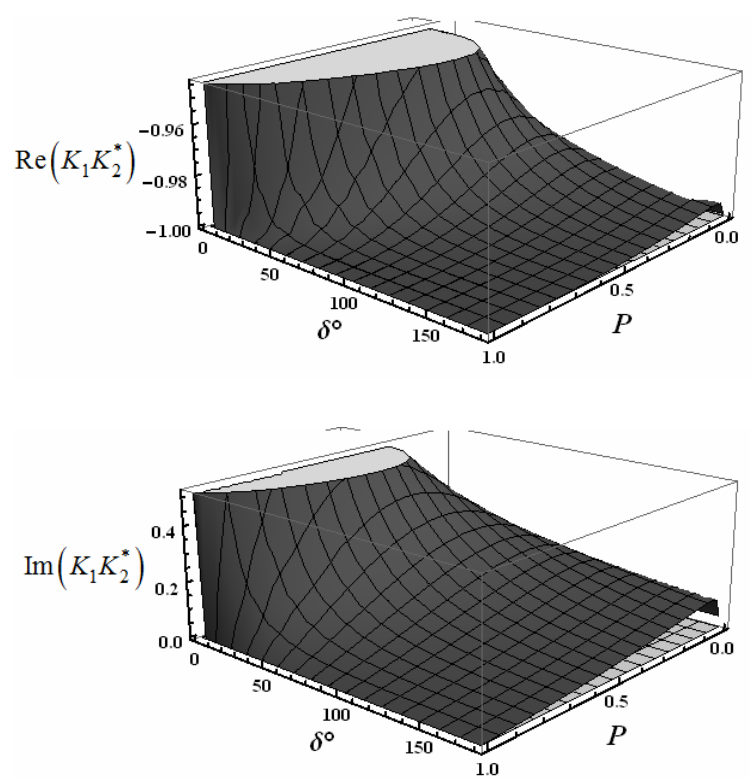

Fig. 3. The product $K_{1} K_{2}{ }^{*}$ for longitudinally inhomogeneous medium with linear birefringence and dichroism at $\theta_{0}=$ $10(\mathrm{deg} / \mathrm{mm})$. The real (a) and imaginary (b) parts of the product $K_{1} K_{2}^{*}$. 

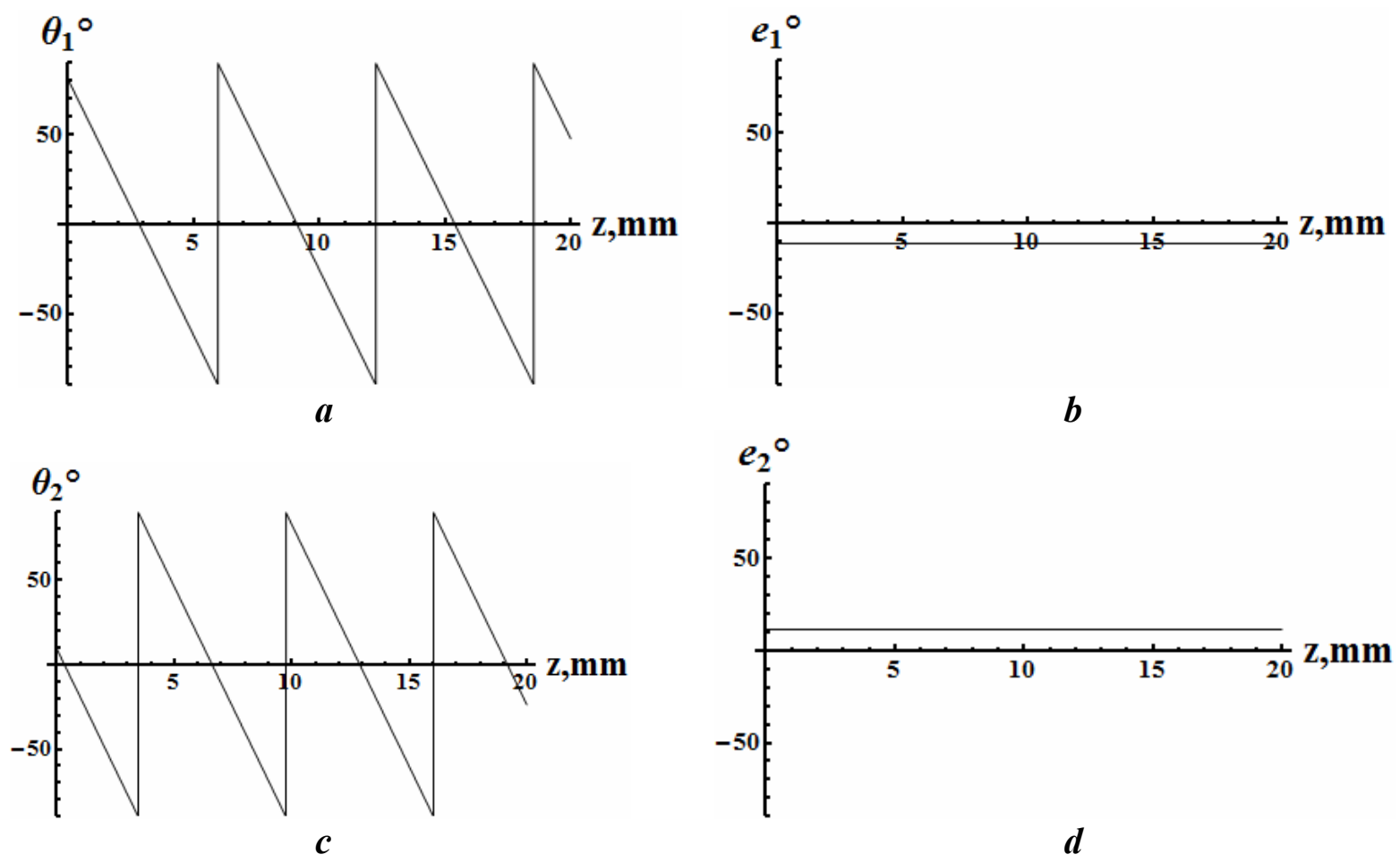

Fig. 4. Changes in the azimuth (a), (c) and angle of ellipticity (b), (d) with the coordinate $z$ along the axis of light propagation in longitudinally inhomogeneous medium with linear birefringence and dichroism and parameters $\xi_{0}=1.22(1 / \mathrm{mm}), \delta_{0}=$ $83(\mathrm{deg} / \mathrm{mm}), \theta_{0}=29(\mathrm{deg} / \mathrm{mm})$.

It implies from Fig. 3 that the polarization states of privileged waves in general are not orthogonal, and orthogonality $\left(\operatorname{Re}\left(K_{1} K_{2}^{*}\right)=-1, \operatorname{Im}\left(K_{1} K_{2}^{*}\right)=0\right)$ is only achieved in the absence of linear dichroism $(P=0)$, which is an obvious result $[8,9]$.

Equation (11) describes evolution of polarization of privileged waves in this class of media. Substituting it into Eqs (13) and (14) $\left(K_{1,2}\right.$ rather than substitute $\left.\theta_{1,2}\right)$, we obtained evolution of polarization parameters: the azimuth and angle of ellipticity at the coordinate $z$ (Fig. 4).

Thus, Fig. 4 implies that these two elliptically polarized privileged waves propagating along the axis of the helical structure $z$ of the inhomogeneous medium "feel" only homogeneous and continuous rotation of the azimuth with distance, and their ellipticity does not change. That is to polarizes $K_{1,2}$, the medium exhibits the effective circular birefringence (optical activity) with the angle of rotation $\varphi=\theta_{0}=\alpha_{0}(\mathrm{deg} / \mathrm{mm})$ and shows no linear birefringence and dichroism. The existence of these waves is due to the features of the structure inherent to the relevant class of media [10] and is the result of converting polarizations of eigenwaves in homogeneous medium with linear birefringence and dichroism, which can be derived from this class of media with $\theta_{0}=0$ (untwisted medium).

\section{Conclusions}

Ascertained in this work is that, in longitudinally inhomogeneous medium with linear birefringence and dichroism, the privileged waves can exist only if coincidence between orientations of corresponding anisotropy axes takes place. Our analysis of polarization parameters of the privileged waves in this case shows that they are non-orthogonal unlike the absence of linear dichroism. Based on the results, we can formulate the problem of synthesis of medium polarization-rotation with settings of the initial ellipticity and azimuth.

\section{References}

1. A.S. Marathay, Matrix-operator description of the propagation of polarized light through cholesteric liquid crystals // J. Opt. Soc. Am. 61, p. 1363-1372 (1971).

2. H. Hurwitz, R.C. Jones, A new calculus for the treatment of optical systems. II. Proof of the three general equivalence theorems // J. Opt. Soc. Am. 31, p. 493-499 (1941).

3. R.C. Jones, A new calculus for the treatment of optical systems. VII. Properties of the N-matrices // J. Opt. Soc. Am. 38, p. 671-685 (1948). 
4. R.M.A. Azzam, N.M. Bashara, Simplified approach to the propagation of polarized light in anisotropic media-application to liquid crystals // $\mathrm{J}$. Opt. Soc. Am. 62, p. 1252-1257 (1972).

5. B. Das, S. Vyas, J. Joseph et al., Transmission type twisted nematic liquid crystal display for three gray-level phase-modulated holographic data storage systems // Opt. Las. in Eng. 47(11), p. 1150-1159 (2009).

6. P. García-Martínez, M. del Mar Sánchez-López et al., Accurate color predictability based on a spectral retardance model of a twisted-nematic liquid-crystal display // Opt. Communs. 284(10-11), p. 2441-2447 (2011).

7. I.S. Kolomiets, S.N. Savenkov, Ye.A. Oberemok, Studying the orthogonalization properties of longitudinally inhomogeneous nondepolarizing media // Registration, data storage and processing, 15(1), p. 23-30 (2013).

8. I.S. Kolomiets, S.N. Savenkov, Ye.A. Oberemok, A.S. Klimov, The solution of the spectral problem for longitudinally inhomogeneous nondepolarizing media // Metallofizika i noveishie tehnologii, 35(9), p. 1197-1208 (2013), in Ukrainian.

9. I.S. Kolomiets, S.N. Savenkov, Ye.A. Oberemok, A.S. Klimov, Studying anisotropic properties of longitudinal inhomogeneous nondepolarizing media with elliptical phase anisotropy // Semiconductor Physics, Quantum Electronics \& Optoelectronics, 16(4), p. 366-373 (2013).

10. S. Chandrasekhar, Liquid Crystals. Cambridge University Press, 1980.

11. R.M.A. Azzam, N.M. Bashara, Elipsometry and Polarized Light. New-York, 1977. 